\title{
versants
}

\section{L'intervista letteraria alla radio. Spunti dagli archivi della RSI}

\author{
Nelly Valsangiacomo \\ Université de Lausanne
}

\begin{abstract}
Il rapporto tra scrittrici e scrittori e la radio risale agli albori di questo media: dizioni, conferenze, corsi, organizzazione culturale, la loro presenza accompagna l'evoluzione della radio, in sintonia con i mutamenti che la cultura e i suoi rappresentanti vivono nello spazio pubblico. Con l'avvento della televisione, un altro possibile luogo di parola si apre. La presenza degli scrittori e, parzialmente, delle scrittrici, è trasversale alle trasmissioni culturali radiotelevisive; questo saggio si interessa però in particolar modo ai diversi aspetti delle interviste letterarie alla radio, partendo da alcuni esempi tratti dagli archivi della Radiotelevisione svizzera di lingua italiana (RSI).

Keywords: intervista letteraria, oralità, genere, posture letterarie, Radio svizzera di lingua italiana (RSI).
\end{abstract}

Catturare e conservare la voce di un personaggio è una delle prime pratiche attuate con la scoperta della registrazione e continua per lungo tempo ad essere uno dei momenti chiave della programmazione radiofonica. L'intervento del personaggio noto e poi l'intervista alla personalità è dunque, in genere, un momento forte: ricordato, ritrasmesso, spesso trascritto e pubblicato. Questo suo valore simbolico, e a volte anche economico, ha permesso la conservazione di questi documenti negli archivi radiotelevisivi, i quali fino ad anni recenti non hanno vocazione patrimoniale, ma solo di produzione. Le voci di protagonisti della scrittura del Novecento noti e meno noti sono dunque ora a disposizione: Benedetto Croce, Hermann Hesse, Vittorio Sereni, Oriana Fallaci o Umberto Eco sfilano nella banca dati della RSI. Una ricchezza documentaria ormai per buona parte accessibile al pubblico, grazie alla banca dati "MMuseo" consultabile nelle biblioteche della Svizzera italiana, ma che va interrogata con prudenza e attenzione. La ricerca per parole chiave deve tenere conto della sedimentazione archivistica e dell'evoluzione dei termini impiegati; e anche la ricerca per nomi propri può essere soggetta a varie problematiche, non da ultimo la semplice sparizione dei documenti, che soprattutto per il periodo precedente agli anni Sessanta, non sono stati conservati, vuoi perché erano trasmissioni in diretta, vuoi perché il costo del supporto di registrazione spingeva al loro riutilizzo.

Molti sono dunque i materiali mancanti, ma la qualità di quelli disponibili merita l'attenzione del mondo della ricerca. E tra questi ricchi spunti, l'intervista è un genere che senz'altro offre molte possibilità di analisi. 
Questo breve saggio vuole proporre alcune piste di indagine sulle interviste letterarie, prendendo le mosse da esempi radiofonici del periodo a cavallo degli anni Settanta del Novecento, nell'intento di offrire spunti complementari, o di supporto, allo studio dettagliato dei singoli interventi di un'autrice o di un autore'.

\section{Gente di lettere e luoghi radiofonici}

Negli ultimi decenni la storia letteraria si è sempre più interessata alle pratiche di creazione e di consumo della produzione letteraria, nutrendo così gli interrogativi della storia culturale. E viceversa. Questo approccio può essere arricchito dall'indagine di uno dei maggiori luoghi di diffusione della letteratura e dei suoi protagonisti nel Novecento: il media radiotelevisivo. L'apertura negli ultimi anni degli archivi aziendali ha permesso al mondo della ricerca di indagare maggiormente in questo ambito, che era stato in un primo tempo abbordato soprattutto attraverso le fonti cartacee.

Se le trasmissioni prettamente letterarie, genere radiotelevisivo in realtà ampio e diversificato, sembrano le più indicate ad indagare questo tema, non va dimenticato che le scrittrici e gli scrittori sono presenti davanti e dietro il microfono dagli albori del mezzo radiofonico, come lo saranno in seguito alla televisione.

La radio in Europa nasce come servizio pubblico, con un mandato fortemente educativo: un contratto di comunicazione pedagogica che determina la scelta dei programmi, elaborati spesso come programmi di cultura umanistica alta, da proporre (o imporre) a un pubblico pensato per lungo tempo dagli organizzatori radiofonici come bisognoso di un'educazione di stampo quasi paternalistico.

Alla ricerca di impieghi che accompagnino la loro attività creativa, i letterati (la ripartizione di genere diventa presto evidente e le donne sono subito, e ampiamente, in minoranza) hanno gioco facile ad imporsi come organizzatori culturali: si pensi a Delio Tessa negli anni Trenta alla RSI o più tardi a Carlo Emilio Gadda alla RAI.

Oltre al loro fondamentale apporto nell'organizzazione, gli scrittori intervengono come specialisti del loro campo, accanto agli studiosi. Dalle prime dizioni e conferenze radiofoniche, passando per i corsi ideati come università popolare, fino alla partecipazione ai dibattiti, la loro presenza nel parlato radiofonico è regolare e significativa.

I Questo saggio riprende e sviluppa alcuni aspetti trattati in una precedente ricerca (Valsangiacomo 20I5). Nelle citazioni si sono omessi i minutaggi, sia per problemi correlati ai diversi supporti di ascolto utilizzati, sia perché si tratta di brevi esemplificazioni. 
Col tempo, l'evoluzione dei generi radiofonici e l'ampliamento degli orari di trasmissione aprono nuovi spazi che ruotano attorno all'attività letteraria e ai suoi protagonisti. La presentazione del libro, unico prodotto a non sottostare alle regole della pubblicità radiofonica, si sviluppa grazie a dispositivi diversificati, mentre l'autore, e sporadicamente l'autrice, assumono un ruolo di rilievo attraverso le interviste letterarie, che definisco come quei momenti di scambio esterni alle trasmissioni sui libri, che danno conto però dell'attività di scrittura e anche dell'opera della persona intervistata, nel suo complesso, o in maniera puntuale.

Fin dagli esordi la radio svizzera di lingua italiana (RSI), come tutte le radio contemporanee, offre uno spazio alle autrici e agli autori o alle loro produzioni: si pensi alla Rassegna letteraria e artistica o alla Mezz'ora culturale, trasmissioni degli anni Trenta poi integrate nelle Cronache culturali. Ma è con l'ampliamento dei programmi radiofonici nel secondo dopoguerra che diverse trasmissioni regolari di presentazione dei libri e, per il nostro caso, di incontro con l'autore, prendono piede.

Le interviste sono sempre più presenti in questi momenti culturali. È per esempio il caso di Terza Pagina, trasmissione di attualità culturale, creata nel I962 che ha, dal 1963, una rubrica dedicata alle interviste, curata da Giorgio Fubiani: Incontri con... La rubrica prevede interviste con personalità pubbliche e della cultura, ma in effetti si occuperà soprattutto di letterati, affiancando diverse altre trasmissioni dedicate esclusivamente a chi i libri li scrive. Sempre alla ricerca di nuove modalità per innovare le trasmissioni, i produttori puntano su un dispositivo di intervista-confessione; la stessa cosa capiterà con Finestra aperta sugli scrittori italiani.

Queste trasmissioni, come quelle che seguiranno, alla radio e alla televisione, meriterebbero un'indagine seriale. Al di là dell'intervento del singolo, che può offrire spunti di maggiore comprensione del personaggio e a volte rivelare utili informazioni sulle pratiche di scrittura e di elaborazione, andrebbe infatti indagato il valore performativo del dispositivo scelto.

\section{L'intervista un genere radiofonico maggiore}

Genere dell'informazione giornalistica, l'intervista accompagna anche la radio sin dagli esordi, negli anni Venti. In seguito, grazie all'evoluzione tecnica, diventa un pilastro del parlato e integra, in alcune occasioni, anche la creazione radiofonica: si pensi alle famose Interviste impossibili (1974-I976), serie di dialoghi tra scrittori viventi e personaggi del passato, trasmessi sul secondo programma della radio italiana, che ebbe grande successo.

È in particolare dagli anni Cinquanta che l'intervista detiene un ruolo importante nell'ambito delle trasmissioni culturali, anche grazie a strumenti quali il magnetofono portatile che permette di raccogliere le voci fuori 
dagli studi e di sfruttare maggiormente la vicinanza di grandi poli culturali, come Milano nel caso della RSI, ma anche la possibilità di intervistare al telefono.

Dal punto di vista formale, gli anni Cinquanta e Sessanta sono anni di transizione, durante i quali si alternano interviste più "paludate", con uno schema molto strutturato in cui giornalisti e scrittori leggono le domande e risposte, ad altre più dirette e spigliate.

Negli anni Sessanta e Settanta, anche grazie alla nuova strumentazione, si può optare per situazioni in cui su una struttura più informale si costruiscono incontri più personali, tentando, a volte anche un po' goffamente, di instaurare un rapporto più immediato per entrare nell'intimità della persona intervistata, che comincia ad interessare non solo nel suo ruolo pubblico, ma anche nei suoi aspetti più personali:

Il microfono ha questo di bellissimo: che può carpire i segreti di un uomo, può facilitarne o addirittura provocarne la confessione senza toccare minimamente l'intimità della sua vita privata [...]. Un uomo davanti al microfono raramente riuscirà a non essere quello che è: ci darà di se stesso un ritratto autentico. $\grave{E}$ col pensiero di raggiungere questa autenticità ritrattistica che la RSI coltiva il piacere degli Incontri diretti².

Questo è quanto affermano nel 1964 i produttori radiofonici della RSI. È un topos che ritorna con molta frequenza quello per cui scrittrici e scrittori si confesserebbero al microfono come in nessun'altra circostanza. In effetti, più che l'essenza umana, che non traspare necessariamente ed esclusivamente attraverso il mezzo radiofonico, i mutamenti dell'intervista ci permettono di indagare altri aspetti, quali la postura pubblica della persona intervistata e soprattutto il suo rapporto con la parola e con l'oralità, alla quale una parte delle personalità del mondo letterario si concede per necessità, poiché il mercato lo impone, mentre altre ne fanno un vero e proprio momento creativo.

Con l'arrivo della televisione, l'intervista alle scrittrici e agli scrittori trova nuovi spazi, almeno fino agli anni Ottanta, periodo in cui si intravvede una certa marginalizzazione, che andrebbe ulteriormente indagata. Resta il fatto che l'intervista nei diversi media diventa uno snodo fondamentale per chi scrive:

La frequenza con cui vengono pubblicate sui giornali interviste più o meno brevi o necessarie sembra avere qualche legame con certi meccanismi del postmoderno: ad esempio, l'inserimento della voce di un autore di letteratura "alta" in un contesto "basso" o midcult [...].

2 La radio 1965: 25. 
Così afferma Niccolò Scaffai (20II: 5) parlando del legame quasi indissolubile tra lo scrittore e i media, che trova un bell'esempio nell'evoluzione dell'intervista letteraria alla radio. Col tempo, infatti, le tipologie dell'intervista si ampliano: da una predominanza dell'intervista-consultazione, nella quale la persona intervistata si esprime soprattutto sul proprio ambito di specializzazione o presenta una propria produzione, si passa a una prevalenza dell'intervista-ritratto, con elementi biografici e personali molto più marcati, e, infine, all'intervista-informazione, momento nel quale l'autore, più che l'autrice, si esprime sull'attualità. I tre tipi convivono a geometria variabile e mutando nel tempo, ma la tendenza alla predominanza del commento socio-politico sulla semplice descrizione del proprio lavoro è evidente.

Se le prime interviste sono strutturate in una stretta forma dialogica di domanda e risposta, col tempo si sviluppano dei dispositivi che mimino la spontaneità del colloquio. Gli elementi propri dell'intervista più personale, anche se a volte solo accennati, caratterizzano inoltre sempre più questo genere radiofonico. Negli anni Sessanta affiora la descrizione fisica, che comincia ad accompagnare con regolarità l'identificazione sociale e geografica della persona intervistata: il «sorriso timido» di Pier Paolo Pasolini intervistato da Giorgio Fubiani (I964), ad esempio. Questa pratica sempre più diffusa, e che mette in parallelo il legame tra intervista e ritratto riscontrato da Ujcich per i giornali (2013: 54), può forse essere indotta dalla presenza sempre più massiccia della televisione quale nuovo media di massa e pertanto dalla necessità quasi mimetica della radio in questo periodo di diventare più "visiva".

Aprendo al vissuto e all'intimità, l'intervista si attualizza lasciando ampi spazi all'immediatezza, se non all'improvvisazione. Anche l'immediatezza prevede però una messa in scena. In questo senso, in alcuni frangenti, il paesaggio sonoro è un vero e proprio attore. I rumori della quotidianità che circonda la persona intervistata ricordano la sigaretta fumata con noncuranza davanti alle telecamere e aiutano nella creazione di un momento più colloquiale e disteso, come si addice a una radio che da «organismo culturale» si vuole ora una «scatola a sorpresa», che elargisce gustose primizie (Marcacci 2009: I68-I69). Così, Ottiero Ottieri è inglobato in un'intervista collettiva nel salotto di casa, durante la quale, con un sottofondo di traffico e squilli del telefono, tutta la famiglia è chiamata ad esprimersi sulla produzione dell'autore 3 .

Gli incisi estranei al tema discusso hanno in questo senso un ruolo importante: «Porti pure via, noi abbiamo finito», dice Mario Soldati al cameriere, durante un'intervista rilasciata nel I972 a Mino Müller (I972). Consi-

3 Intervista curata da Alfredo Barberis, I6.I2.1972. 
derare questi aspetti nell'analisi dell'intervista permette di comprendere pienamente il senso e le parole del dialogo, arricchendo la conoscenza del personaggio e il ruolo sociale che gli si riconosce.

Questo cambiamento presuppone non solamente nuove competenze radiogeniche della persona intervistata, comprese le capacità, al contempo di postura e di fiction, fondamentali per la gente di cultura che vuole mettersi in scena (Jaeggle 2007), ma anche l'abilità della persona che intervista di far emergere tali qualità. Interrogarsi sulla figura e sul ruolo del giornalista è difatti fondamentale per capire i meccanismi dell'intervista. All'inizio il giornalista culturale non è ancora circondato da quell'aura di credito intellettuale che acquisisce in seguito, per cui generalmente si pone in posizione subalterna rispetto all'intervistato. Col tempo, il rapporto tra intervistatore e intervistato si riequilibra e porta a volte all'utilizzo del tu e a forme altamente colloquiali e aperte, non sempre riuscite peraltro dal punto di vista del rapporto tra chi intervista e chi è intervistato.

\section{Tra generi e genere}

La radio, pur aprendo a nuovi spazi di creazione, riproduce per certi versi le gerarchie del mondo culturale. Considerate le diverse variabili, quali la disponibilità delle persone o la loro capacità a esprimersi al microfono, questo fenomeno è particolarmente visibile se ci si riferisce alla quantità e agli ambiti di intervento delle scrittrici, e più in generale alla presenza delle donne nelle trasmissioni del parlato, per lungo tempo chiaramente minoritaria o confinata a trasmissioni specifiche.

Gli aspetti di genere emergono anche nei contenuti delle trasmissioni e nei dispositivi dell'intervista stessa. La donna di cultura intervistata è sollecitata spesso su argomenti di costume, ai quali rischia poi di rimanere ancorata la sua immagine; e questo non solo nel mondo radiofonico; un aspetto che è chiaramente espresso da Camilla Cederna al microfono della RsI:

Certo su un giornale noioso com'era nei primi tempi l'Europeo l'unica donna che c'era era condannata a fare una rubrica che si chiamava proprio società [...]. Però anche durante questi periodi io ho fatto delle cose serie che la gente non si ricorda mai [...] alcuni giorni fa degli amici di Lugano parlando con degli amici di Milano hanno chiesto: cos'è successo alla Cederna che da così mondana è diventata di colpo impegnata? (Canitano Aragno I970).

La voglia dichiarata di mostrare le diverse sfaccettature della propria attività, evitando ogni semplificazione, non è che parzialmente accolta dalla collega che la intervista, Anna Aragno, la quale, pur affrontando aspetti forti della carriera della Cederna, torna ad insistere sullo sguardo frivolo, 
indagando l'intimità della scrittrice con domande connotate tra il femminile (perché non si è sposata? e cosa pensa della moda?) e il femminista (qual è la sua opinione sul divorzio?). Questo all'interno di una rubrica, gli Incontri, che in qualche modo consacra i personaggi famosi, ma che al contempo, tramite la struttura delle interviste, gerarchizza la loro importanza simbolica.

In generale, le donne intervistate sembrano dover in qualche modo rappresentare la loro categoria di genere più di quanto accada per gli uomini, i cui interventi vertono o sulla loro produzione o su questioni più ampie di società e cultura. È un aspetto che andrebbe analizzato con una riflessione intersezionale, che consideri in senso più ampio l'immagine mediale delle minoranze, spesso rappresentate in una visione generalizzante, che offre poco spazio all'individualizzazione.

Anche quando si discute della produzione della protagonista e sono introdotti aspetti di success story, gli elementi di una costruzione di genere sono chiaramente riconoscibili. Oriana Fallaci, che «non è una moderna suffragetta tutta pepe e parolacce», riceve Giancarlo Meda (197I) in un albergo a Milano ${ }^{4}$ «stesa sul letto per un vago malore», descrizione che assume un forte valore connotativo.

La dualità tra atteggiamenti considerati virili o femminili della Fallaci pervade tutta l'intervista, da ambedue le parti. La scrittrice sente di doversi posizionare rispetto ai colleghi uomini, cosa che farà lungo tutta l'intervista insistendo sia sulla sua intesa maggiore con questi ultimi («La donna deve fare il lavoro come un uomo ma un poco meglio, ma io non mi sono mai messa in rivalità con loro»), sia sulla sua posizione particolare di donna nel mondo della scrittura giornalistica («Io non vengo come la maggior parte delle donne che fanno del giornalismo, dalla cronaca mondana o dai servizi rosa. Io vengo dalla cronaca nera»).

Una presa di distanza da un mondo di giornaliste un po' stereotipato, che non manca però di mostrare difficoltà e ambiguità, come dimostra la sua riflessione sul successo:

Il successo grosso è esploso dopo i servizi nel Vietnam, perché una donna con una pentola in testa ha un altro effetto sulla gente. Io credo che l'intelligenza sia neutra, però una donna alla guerra porta una visione nuova di fatti, che non ha niente a che fare con il punto di vista femminile di cui parlano i direttori dei giornali... un punto di vista insultante. Non riesco a concepire come ci siano delle pagine dedicate agli uomini e delle pagine dedicate alle donne. Però porta un elemento nuovo, in quanto la guerra non è un suo elemento naturale e dunque la guarda con occhi diversi. Io l'ho guardata con occhi diversi.

4 Le citazioni seguenti di Fallaci si riferiscono tutte a questa intervista. 
In questo caso, l'impostazione stessa dell'intervista non aiuta a superare le ambivalenze, anzi conforta una visione con connotazioni di genere.

\section{La corporeità nell'intervista}

Al contrario delle trasmissioni dedicate alla presentazione dei libri, nell'intervista letteraria l'opera scritta tende a scomparire di fronte all'importanza dell'intervista stessa, che diventa la forma per eccellenza dell'intervento pubblico di chi scrive e che per certi versi basta a se stessa (Martens-Meurée 20I4). Se la messa in scena del libro è il cuore di trasmissioni specifiche, non sempre in presenza dell'autore, nelle interviste letterarie il libro è uno spunto, quasi un pretesto, per avvicinare la persona nelle sue diverse sfaccettature: si incontra il personaggio, che è tale grazie alla sua opera, ma paradossalmente l'opera perde d'importanza, a favore del contesto di produzione della stessa.

L'intervista a Maria Corti, condotta da Alfredo Barberis (1972), permette di riprendere i diversi livelli di analisi fin qui citati e abbordarne un ultimo: quello dell'importanza, nello studio della fonte radiofonica, di porre l'attenzione anche alla corporeità che emerge dall'intervista, intesa qui come la presa in considerazione della voce, che è il vero medium forte dell'intervista radiofonica (Karpf 20II; Le Breton 20II).

Come per Fallaci, già nel cappello introduttivo Corti è presentata come un caso anomalo nel mondo delle scrittrici. Di cattedratici che siano anche scrittori in proprio ve ne sono molti, afferma Barberis, ma «di professoresse universitarie che siano anche romanziere e vincano coi loro libri premi letterari ne conosciamo soltanto una: Maria Corti».

È dunque spesso l'eccezionalità che viene sottolineata per giustificare la presenza di una scrittrice nel consesso delle interviste letterarie. Barberis gioca all'inizio su questa eccezionalità: «Cosa pensa la Corti accademica della romanziera Corti, come collocarla storicamente nell'alveo della letteratura italiana?».

La risposta articolata della Corti, che si dilunga sul primo romanzo, L'ora di tutti (1962), è interessante non solo per il contenuto, ma anche per come la figura della scrittrice si intravvede attraverso il ritmo della parola e l'impostazione della voce. La dettagliata spiegazione sui modi di fare letteratura - il primo, quello eversivo, dell'avanguardia; il secondo, che prevede di continuare come se nulla fosse accaduto; il terzo, cercare una via che recuperi i generi letterari del passato con una nuova chiave interpretativa, nel quale Corti si situa - assume pienamente il suo significato se si tengono presenti il ritmo della parola, i toni e le modulazioni della voce. Corti propone delle descrizioni succinte, cui si aggiungono brevi commenti molto più veloci, a sintetizzare i suoi propositi. È una voce sicura e ferma, il cui tono si alza 
e diventa squillante per sottolineare gli aspetti che più le premono, come quando si attarda nello spiegare come a dieci anni di distanza il libro sia maggiormente compreso dalla critica:

Evidentemente dieci anni hanno fatto bene a questo libro [la voce sorride] [...]. La critica oggi lo ha capito abbastanza bene [la voce si alza]. In fondo è l'operazione che poi ho fatto, più vistosamente, con Il ballo dei sapienti [la voce torna più pacata], come lei giustamente ha detto.

Si ritrova la stessa modalità vocale quando parla del fondo Fenoglio, sul quale sta lavorando con gli allievi:

Poi ogni tanto partiamo, con una o due macchine e andiamo a passare qualche giornata ad Alba [...] sono giornate che permettono di lavorare in loco [...]. La sera finito il lavoro, abbiamo l'abitudine di trovarci con i vecchi amici di Fenoglio.

Così anche quando narra del suo lavoro a Recanati sugli inediti di Giacomo Leopardi. Nella voce si sentono la sicurezza di un terreno conosciuto, quello della ricerca, la pacatezza della competenza e la passione per quello che si sta compiendo:

Mi permetta, giacché ho il microfono a disposizione, di dire una cosa. Come era più civile quest'epoca nel senso che c'era la civiltà di provincia. In Italia, e probabilmente anche in altre nazioni, cittadine di quindici, ventimila abitanti avevano delle meravigliose biblioteche, erano meravigliosi centri di studio.

Nemmeno alcune domande trabocchetto di Barberis riescono a smuoverla.

Il discorso è tutt'altro quando si entra in quella parte di intervista che prevede domande più personali, ma per alcuni versi spersonalizzanti (pregi e difetti, viaggi o pittori preferiti) sulla falsa riga del questionario di Proust, utilizzato in quel periodo anche nella famosa trasmissione culturale francese Apostrophes di Bernard Pivot.

Il giornalista obbliga Corti a uscire dalla sua postura privilegiata di scrittrice e ricercatrice, per entrare in aspetti che pur essendo anche di ordine culturale, la imbarazzano, la rendono tentennante, fino quasi a scocciarla, mentre il giornalista incalza: qual è il suo attore teatrale preferito? crede che la sua scrittura sia stata influenzata da un'arte visiva? Ed altri quesiti di questo tenore. Sono le pause, le risposte secche, i tentennamenti, così come l'incapacità del giornalista di entrare in empatia con l'intervistata, che permettono di carpire le informazioni maggiori non solo sulla persona di Corti, 
ma anche sulle aspettative nei confronti della parola in pubblico: la parola e la voce sono definite da uno spazio e un tempo condivisi e nemmeno la mediazione del microfono e dell'eventuale rimaneggiamento riescono a rassicurare la persona intervistata. È un modo diverso di comunicare rispetto a quello di una scrittura lenta e ripetuta:

Io sono enormemente arcaica, scrivo a mano con penna, spesso nemmeno con biro, addirittura con la vecchia penna stilografica. Uso la macchina quando sono arrivata perlomeno alla penultima stesura.

E nel descrivere questa operazione così rituale, Corti ritrova la voce ferma, che le permette di aprire degli spiragli sulla sua vita di scrittrice:

Ho bisogno di silenzio assoluto. Mi sono disperata per trovare a Milano una casa da cui non sentissi nemmeno il motore di un'automobile.

Confessione che permette al giornalista di descrivere all'ascoltatore il luogo dell'intervista, la «bella casa di Maria Corti [che] dà su un giardino, uno dei pochi giardini milanesi, e il suo studio si affaccia sul verde, adesso autunnale». Il tono più colloquiale, con accenni di pudore, è infine destinato a Pellio, in Val d'Intelvi, suo luogo d'origine:

È una zona che amo molto perché è una zona di confine [...]. Le zone di confine sono, come dire, polisemantiche, sono ambigue [...]. Risentono di un doppio mondo [...]. Nella popolazione locale io vedo caratteristiche italiane e vedo caratteristiche svizzere.

Sono solo brevi stralci di una lunga intervista, ma che vorrebbero mostrare l'utilità di non dimenticare il supporto, anche quando si lavora sulle fonti radiofoniche. Solo operando in questo modo si può dare parzialmente ragione agli organizzatori culturali della radio quando affermavano, come citato nelle prime pagine: «Un uomo davanti al microfono raramente riuscirà a non essere quello che è: ci darà di se stesso un ritratto autentico»5. Questa restituzione avviene tuttavia tramite l'ascolto dell'oralità: è l'utilizzo della voce e della parola, e non il semplice contenuto che ci parla. Così come il rapporto tra testo e immagine è una totalità da indagare, nel caso della fonte radiofonica solo la relazione tra oralità, parola e significato rende il contenuto nella sua interezza.

Va ovviamente tenuto conto di come e quanto il medium influenzi questi aspetti. Non va infatti dimenticato che il mutamento dell'estetica radiofoni-

5 Cfr. nota 2. 
ca, in parallelo con l'evoluzione tecnica, ha sempre modificato il dialogo preregistrato e toglie ormai parte dei significanti originari. Ma qualche accenno rimane sempre, anche nella più invasiva delle ripuliture, proprio perché il confronto con un dispositivo non è mai neutro.

\section{Oralità e scrittura: l'intervista come genere letterario}

L'oralità ha sempre fatto parte integrante del mestiere della scrittura (Waquet 2003): si tengono conferenze, si presentano libri. La radiofonia riproduce una pratica preesistente. Alla radio si tengono brevi conferenze, si parla di libri, leggendone a volte degli estratti; si declamano, infine le proprie poesie. Questa sempre più importante attività orale, determinata da una forte esigenza di mediatizzazione suscita non poche perplessità. Come nel caso di Franco Fortini, che nella trasmissione Selva letteraria sosteneva non fosse opportuno leggere a voce alta una poesia quasi sempre scritta per una lettura mentale (Fortini I998). In un contesto più ampio Jan Baetens riflette criticamente sul quello che sembra essere ormai un paradigma dell'attualità letteraria: lo scrittore deve leggere in pubblico la sua opera: senza la parola, la scrittura sembra ormai scomparire (Baetens 20I6: 9).

Si tratta di una tendenza che sembra capovolgere per certi versi quello che è stato per lungo tempo: le conferenze, i convegni, gli incontri si completavano nella pubblicazione di un testo, l'unica a poter consolidare il riconoscimento di quanto proposto. Anche la radiofonia partecipa dai suoi albori a questa pratica. I conferenzieri più famosi vedevano il loro testo stampato nel giornale della radio: fu così, come per molti altri, per Benedetto Croce quando tenne il suo unico intervento radiofonico alla RSI nel I936 (Valsangiacomo 20I5: 37). Gli interventi erano regolarmente ripresi e ripubblicati poi dagli stessi conferenzieri. Spesso anche l'intervista segue questo percorso, al punto che, nella forma del libro-intervista, l'intervista è ormai considerata un genere letterario. Per alcuni, questa nuova tipologia di pubblicazione sarebbe scaturita dall'intervista radiofonica, genere giornalistico che avrebbe predisposto il pubblico a un nuovo consumo (Lejeune I980).

È certo che la trascrizione, più o meno fedele, delle interviste è una pratica corrente nel mondo della radio. Travalicando l'interesse del contenuto, è spesso correlata alla fama del personaggio - e dunque per estensione a quella del giornalista che lo intervista - e alla sua predisposizione a questo tipo di dialogo. Nel caso della RSI, gli esempi sono diversi e spaziano dalle raccolte di interviste, come nel caso di Incontri con scrittori svizzeri di Claudio Nembrini (1977), a Eugenio Montale, scrittore con una certa simpatia per questa modalità di intervento, ricambiato dai giornalisti che in diverse occasioni hanno riprodotto le sue interviste. Il suo caso, inoltre, è significativo del diverso tipo di trascrizioni possibili: dall'utilizzo di passi non trasmessi 
alla radio, alla proposta di alcuni spezzoni, fino a una trascrizione che prevede un'ampia libertà interpretativa rispetto all'originale. Laddove sia possibile, il confronto con l'originale apre nuove piste di studio sul rapporto tra oralità e scrittura.

Per concludere, la parola pubblica è un elemento fortemente presente nella vita di chi ha fatto della scrittura la sua professione. Per lungo tempo però non ha lasciato che sporadiche tracce. L'intervista letteraria alla radio e alla televisione permette di confrontarsi con questa parola, spesso poco considerata, quando non deprezzata. La sua analisi, che merita un approccio interdisciplinare, oltre ad offrire nuovi elementi di conoscenza sui protagonisti, permette di aggiungere allo studio della composita categoria dei letterati un ulteriore elemento: quello dell'oralità e del suo rapporto con la scrittura.

\section{Bibliografia}

Baetens, Jan, A voix haute. Poésie et lecture publique, Bruxelles, Les Impressions Nouvelles, 2016.

Fortini, Franco, Breve secondo Novecento, prefazione di R. Luperini, note di L. Lenzini, Lecce, Manni, I998.

Jaeggle, Claude, L'interview. Artistes et intellectuels face aux journalistes, Paris, Puf, 2007.

Karpf, Anne, The Human Voice. The Story of a Remarkable Talent, London, Bloomsbury Publishing, 201 .

La radio della Svizzera italiana nel millenovecentosessantaquattro, [s.n.t.], consultato in Radiotivu, I8.4.1965.

Le Breton, David, Éclats de voix. Une anthropologie des voix, Paris, Métailié, $20 I I$.

Lejeune, Philippe, Je est un autre. L'autobiographie de la littérature aux médias, Paris, Seuil, I980.

Martens, David - Meurée, Christophe, Secrets d'écrivains. Enquête sur les entretiens littéraires, Bruxelles, Les Impressions Nouvelles, 2014.

Marcacci, Marco, «L'avvento della televisione e il rinnovamento della radio (I962-I982)», in Th. Mäusli (a cura di), Voce e specchio. Storia della radiotelevisione svizzera di lingua italiana, Locarno, Dadò, 2009, pp. I2I-I96.

Nembrini, Claudio, Incontri con scrittori svizzeri. Fringeli, Marti, Muschg, Bichsel, Federspiel, Steiner, Jotterand, Chessex, Borgeaud, Voisard, Grobéty, Bellinzona, Casagrande, I977.

Scaffai, Niccolò, «L'intervista con l'autore: il caso Montale», in I. Crotti et al. (a cura di), Autori, lettori e mercato nella modernità letteraria, Pisa, ETS, 2OII, II, pp. 9-I8. 
Ujcich, Veronica, L’intervista. Tra giornalismo e letteratura. Alberto Moravia, Pier Paolo Pasolini, Primo Levi, Roma, Aracne, 2013.

Valsangiacomo, Nelly, Dietro al microfono. Intellettuali italiani alla radio svizzera (1930-1980), Bellinzona, Casagrande, 2015.

Waquet, Françoise, Parler comme un livre. L'oralité et le savoir (XVIe-XXe siècles), Paris, Albin Michel, 2003.

\section{Indice delle interviste}

Barberis, Alfredo (a cura di), «Incontro con Ottiero Ottieri», in [RsI] Finestra aperta sugli scrittori italiani, I6.I2.1972.

Barberis, Alfredo (a cura di), «Incontro con Maria Corti», in [RSI] Finestra aperta sugli scrittori italiani, 23.12.1972.

Canitano Aragno, Anna (a cura di), «Camilla Cederna», in [RSI Incontri, 7.IO.1970.

Fubiani, Giorgio (a cura di), «Incontro con Pier Paolo Pasolini», in [RSI] Terza pagina, 5.2.1964.

Meda, Giancarlo (a cura di), «Oriana Fallaci scrittrice», in [RsI] Incontri, 22.2.1971.

Müller, Mino (a cura di), «Mario Soldati», in [RSI] Incontri, 18.9.1972. 